\title{
Acute Aortic Syndrome in Marfan Syndrome with Chest Pain and Negative Initial Imaging
}

\author{
Sorush Rokui ${ }^{1}$, Edward Percy ${ }^{1}$, and Joel Price ${ }^{1}$ \\ ${ }^{1}$ The University of British Columbia
}

September 25, 2021

\begin{abstract}
A 25-year-old male with Marfan Syndrome and previous aortic root surgery presented with severe chest and upper back pain. Initial CT angiogram showed mild aortic dilatation but no acute dissection. Anti-impulse therapy was initiated. Five days after presentation, follow-up CT angiography revealed a new pseudoaneurysm of the descending thoracic aorta. The patient underwent replacement of the mid-descending thoracic aorta with a collagen-impregnated woven polyester tube graft without complication. Despite negative initial imaging and relatively small aortic size, patients with Marfan Syndrome with acute chest pain should be treated with high suspicion and may benefit from admission and serial imaging.
\end{abstract}

Acute Aortic Syndrome in Marfan Syndrome with Chest Pain and Negative Initial Imaging.

Brief title: MFS-related Aortic Syndrome.

Sorush Rokui MD ${ }^{1}$, Edward D. Percy MD MSc${ }^{1}$, Joel Price MD, MPH ${ }^{1}$

${ }^{1}$ Division of Cardiac Surgery, University of British Columbia, Vancouver, Canada

\section{Address for Correspondence:}

Dr. Joel Price Division of Cardiac Surgery, University of British Columbia 494-1081 Burrard Street, St. Paul's Hospital Vancouver, British Columbia, Canada V6Z 1Y6 Phone: +1 (604) 806-9307 Email: joel.price@vch.ca

\section{Sources of funding: None.}

Keywords: aorta and the great vessels, cardiovascular pathology, cardiovascular research.

\section{Abstract}

A 25-year-old male with Marfan Syndrome and previous aortic root surgery presented with severe chest and upper back pain. Initial CT angiogram showed mild aortic dilatation but no acute dissection. Anti-impulse therapy was initiated. Five days after presentation, follow-up CT angiography revealed a new pseudoaneurysm of the descending thoracic aorta. The patient underwent replacement of the mid-descending thoracic aorta with a collagen-impregnated woven polyester tube graft without complication. Despite negative initial imaging and relatively small aortic size, patients with Marfan Syndrome with acute chest pain should be treated with high suspicion and may benefit from admission and serial imaging.

\section{Introduction}

Marfan syndrome (MFS) confers a significant risk of aortic aneurysm and dissection. This is reflected in lower thresholds for surgical management of aortic pathology ${ }^{1}$. Stanford type B aortic dissections in this population occur frequently in patients with previous aortic surgery and are more often treated surgically 
compared to the non-MFS population ${ }^{2}$. We present the case of an insidious acute aortic syndrome that highlights the seriousness and variability of pain as a presenting symptom of acute aortic syndrome in MFS. Ethics approval was not required for this study, and informed consent of the patient described was obtained.

\section{Case Description}

A 25-year-old male patient with a height of $203 \mathrm{~cm}$ presented to the emergency department with severe chest and upper back pain. Relevant history includes a diagnosis of MFS with confirmed FBN1 gene mutation. Two years earlier, he underwent successful aortic valve-sparing root replacement using the reimplantation technique for an aneurysmal aortic root. He presented with similar symptoms. At the time of initial surgery, an incidental, chronic-appearing dissection of the ascending aorta was noted.

On present examination, there was moderate, symmetrical bilateral hypertension and no evidence of malperfusion. Although acute aortic syndrome was suspected, computed tomography angiography (CTA) revealed no evidence of aortic dissection or rupture, showing only dilatation of the mid descending thoracic aorta from $33 \mathrm{~mm}$ to $37 \mathrm{~mm}$ within 24 months (Figure 1a ). There was also a stable, benign appearing peri-graft collection of the ascending aorta unchanged from prior imaging. Repeat CTA within 12 hours of presentation demonstrated no change. Nonetheless, given the history and lack of an alternative diagnosis, the patient was admitted, and anti-impulse therapy was initiated.

The patient's course in hospital was relatively benign, with improvement, but not complete resolution, of his pain. Given the stable nature of the clinical picture, discharge was considered. A pre-discharge repeat CTA demonstrated progressive dilatation of the descending thoracic aorta to $40 \mathrm{~mm}$, as well as an intraluminal flap with adjacent crescent-shaped contrast-filled lesion consistent with a new, localized type B aortic dissection or pseudoaneurysm (Figure 1b ). Given this new lesion despite anti-impulse therapy, the decision was made to operate on the patient.

The patient was brought to the operating room as an urgent case. Left thoracotomy was performed at the fifth intercostal space. Aortic clamp sites were isolated proximally, immediately distal to the left subclavian artery, and distally at the mid-descending thoracic aorta. Left heart bypass was then initiated via an $8 \mathrm{~mm}$ chimney graft on the left common femoral artery, and the left inferior pulmonary vein.

The aorta was then clamped and incised longitudinally. This revealed a longitudinal defect in the posterior wall of the aneurysmal portion of the aorta (Figure 2 ). The aorta was divided proximally and distally, and pairs of bleeding intercostal arteries were oversewn. A $20 \mathrm{~mm}$ collagen-impregnated woven polyester tube graft was selected for repair. Both proximal and distal anastomoses were sewn with 3-0 polypropylene suture in continuous fashion and PTFE felt externally.

There were no intraoperative complications. The postoperative course was relatively unremarkable, and the patient was discharged on postoperative day six.

\section{Comment}

The aortic event rate in patients with MFS is, at least in part, a function of aortic size ${ }^{1,3}$. Patients with MFS tend to present earlier in life, and often receive more than one aortic intervention ${ }^{2}$. A large retrospective analysis followed 600 MFS patients longitudinally, and this study identified prior prophylactic aortic surgery and proximal descending thoracic aortic diameter at or greater than $27 \mathrm{~mm}$ as variables independently predictive of Type B dissection ${ }^{4}$.

This case report highlights the important clinical lesson that pain as the presenting symptom merits serious concern in patients with MFS, even in the setting of sub-operative aortic diameter and negative initial imaging. In this population, who can experience acute aortic syndromes at relatively small diameters ${ }^{4}$, there may be an increased sensitivity and specificity for pain without other explanation in the diagnosis of acute or impending aortic events. Exercising a low threshold for admission or close follow-up of MFS patients with thoracic pain, in conjunction with serial imaging, may reveal acute aortic pathology of atypical clinical 
course. This may be particularly true in MFS patients with previous aortic surgery or known aneurysmal disease. A high index of clinical suspicion is prudent in these patients.

\section{Author contributions}

All three authors of this manuscript provided substantial contribution to the concept, structure, drafting, revision, and approval of this manuscript.

\section{References}

1. Hiratzka LF, Bakris GL, Beckman JA, et al. 2010 ACCF/AHA/AATS/ACR/ASA/SCA/SCAI/SIR/STS/SVM guidelines for the diagnosis and management of patients with Thoracic Aortic Disease: a report of the American College of Cardiology Foundation/American Heart Association Task Force on Practice Guidelines, American Association for Thoracic Surgery, American College of Radiology, American Stroke Association, Society of Cardiovascular Anesthesiologists, Society for Cardiovascular Angiography and Interventions, Society of Interventional Radiology, Society of Thoracic Surgeons, and Society for Vascular Medicine. Circulation. 2010;121(13):e266-369.

2. de Beaufort HWL, Trimarchi S, Korach A, et al. Aortic dissection in patients with Marfan syndrome based on the IRAD data. Ann Cardiothorac Surg. 2017;6(6):633-41.

3. Jondeau G, Detaint D, Tubach F, et al. Aortic event rate in the Marfan population: a cohort study. Circulation. 2012;125(2):226-32.

4. den Hartog AW, Franken R, Zwinderman AH, et al. The risk for type B aortic dissection in Marfan syndrome. J Am Coll Cardiol. 2015;65(3):246-54.

\section{Figure Legends}

Figure 1. Pre-operative imaging of suspected aortic dissection. A) Negative CT angiography demonstrating no dissection or rupture of the thoracic aorta, with dilatation of the thoracic aorta to $37 \mathrm{~mm}$. B) Repeat CT angiography five days later, demonstrating new contrast-filled crescentic defect.

Figure 2. Intra-operative photograph of longitudinal defect in the descending thoracic aorta after left thoracotomy and replacement.

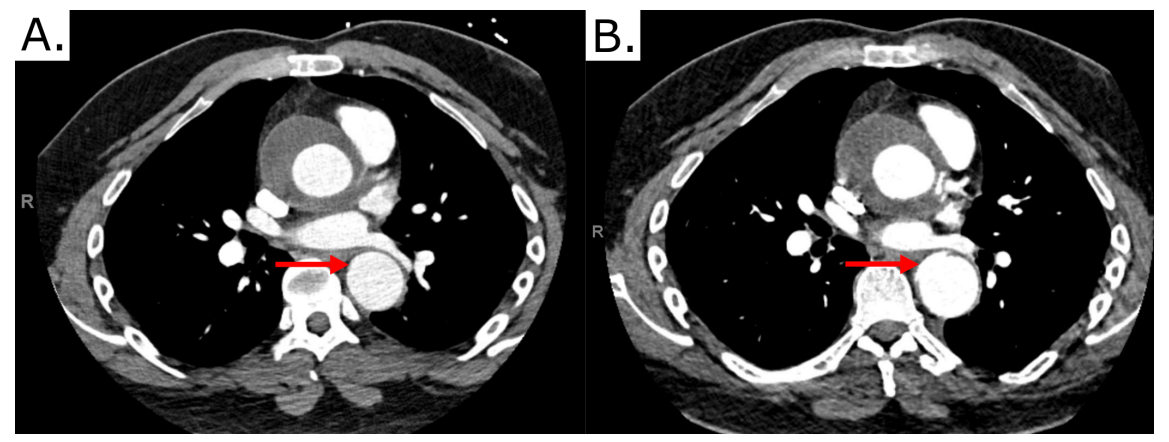




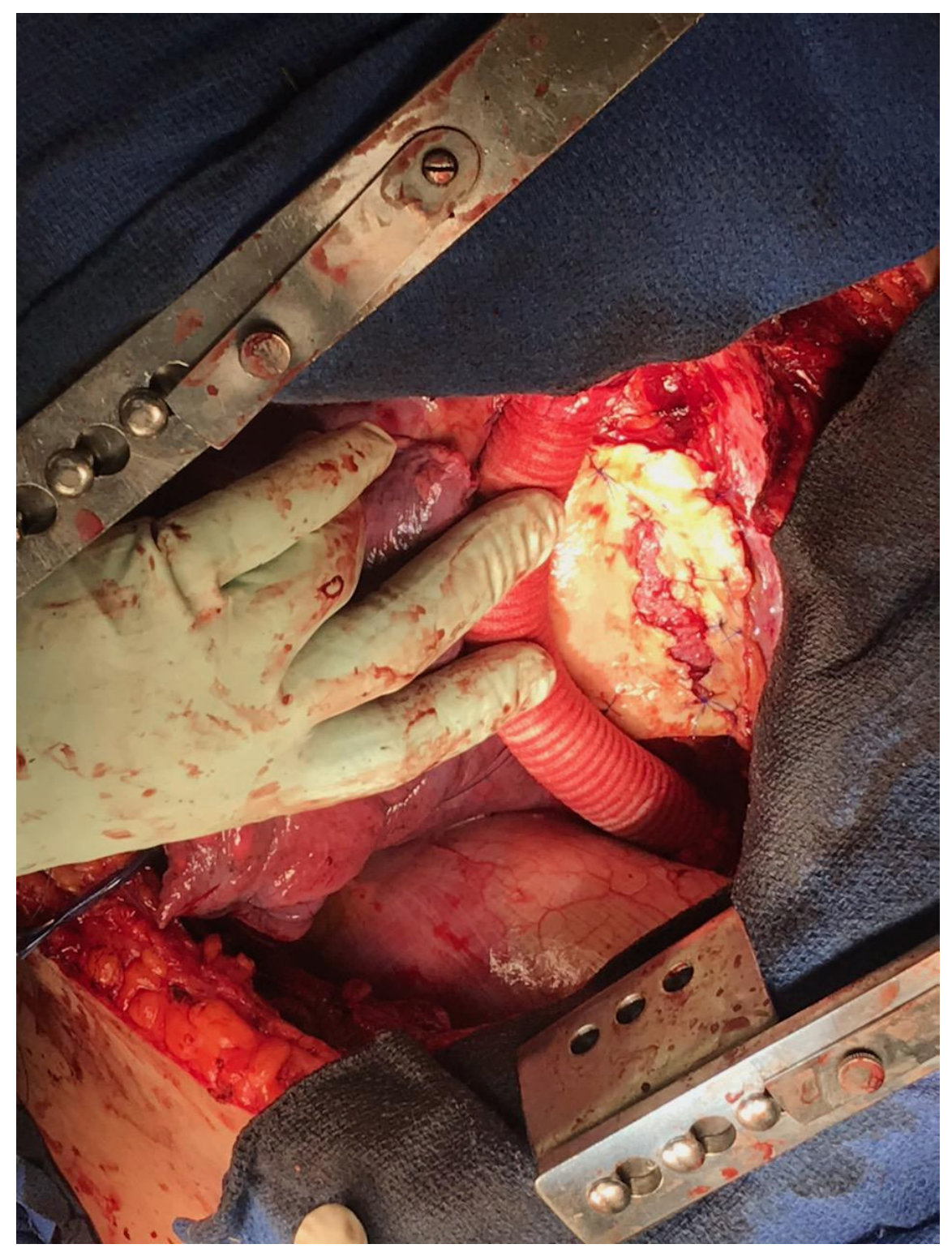

